\title{
Topical lignocaine anaesthesia for oropharyngeal sampling for COVID-19
}

\author{
Anupam Kanodia ${ }^{1}\left(\mathbb{D} \cdot\right.$ Deepankar Srigyan $^{2} \cdot$ Kapil Sikka $^{1}\left(\right.$ D $\cdot$ Aashish Choudhary $^{2} \cdot$ Avinash Choudekar $^{2}$. \\ Saurabh Mittal ${ }^{3} \cdot$ Shweta Arun Bhopale ${ }^{4} \cdot$ Lalit Dar $^{2} \cdot$ Alok Thakar $^{1}$
}

Received: 26 August 2020 / Accepted: 23 September 2020 / Published online: 1 October 2020

(c) Springer-Verlag GmbH Germany, part of Springer Nature 2020

\begin{abstract}
Objectives To ascertain if topical lignocaine application in oropharynx prior to swab sampling to test for COVID-19 improves a patient's comfort and to assess its effect on the swab sample taken to conduct the RT-PCR.

Methods Adult patients testing positive on the RT-PCR COVID-19 test were sampled again within $48 \mathrm{~h}$ after administering topical oropharyngeal anaesthesia. Patients were asked to rate their discomfort on a visual analog scale (VAS) for both sample A and B. A qualitative real-time RT-PCR for detection of SARS-CoV-2 RNA, was performed, and the cycle threshold value $(\mathrm{Ct})$, used as a surrogate marker for the viral load, was measured for the sample taken without lignocaine (sample A) and the sample taken post-lignocaine application (sample B). The difference in $\mathrm{Ct}$ values of both the groups was checked for any statistical significance using paired t-test. Wilcoxon signed rank test was used on VAS scores to determine any significant decrease in discomfort.

Results Forty patients were included in the study. Twenty-nine patients (72.5\%) reported the procedure to be more comfortable post-lignocaine application. Median (IQR) discomfort on VAS decreased from 7 (1) to 5 (2) after lignocaine use, which was statistically significant $(p<0.05)$. Mean Ct value for sample A was $17.21 \pm 5.25$ and for sample B was $18.44 \pm 4.8$ $(p>0.05)$, indicating a non-significant effect of lignocaine on SARS-CoV-2 concentration in the sample.

Conclusion Topical lignocaine, while improving the comfort of the procedure of oropharyngeal sampling for patient did not alter the SARS-CoV-2 viral load that was detected in nasal and oropharyngeal samples taken together.
\end{abstract}

Keywords Lignocaine $\cdot$ SARS coronavirus $\cdot$ RT-PCR

\section{Introduction}

Real Time reverse-transcription polymerase chain reaction (RT-PCR) performed on adequately swabbed nasal and oropharyngeal tissue is considered the gold standard for diagnosing SARS-Coronavirus-2 (CoV-2) infection [1]. One of

Kapil Sikka

kapil_sikka@yahoo.com

1 Department of Otorhinolaryngology and Head- Neck Surgery, Room No 4057, All India Institute of Medical Sciences, Ansari Nagar, New Delhi, Delhi 110029, India

2 Department of Microbiology, All India Institute of Medical Sciences, New Delhi, India

3 Department of Pulmonary, Critical and Sleep Medicine, All India Institute of Medical Sciences, New Delhi, India

4 Department of Oncoanesthesia and Palliative Medicine, All India Institute of Medical Sciences, New Delhi, India the major challenges while sampling is the gag and cough reflex that leads to droplet (5-10 $\mu \mathrm{m}$ particles) expulsion and aerosol $(<5 \mu \mathrm{m}$ particles) generation [2]. These reflexes pose an additional risk to the health care workers (HCWs) involved in sampling. It is also possible that the consequent inadequate or "defensive" sampling leads to false negative tests.

Otolaryngologists routinely employ lignocaine lozenges to minimise the gag reflex while performing diagnostic and therapeutic procedures in the oral cavity and oropharynx. The preparations are generally well tolerated, safe and effective. The current study was conducted on the premise that lignocaine reduces patient and physician discomfort and objectively evaluated for any improvement in patient's comfort while sampling. The theoretical possibility of lignocaine or its preservatives compromising the viability of the virus or its components, e.g. nucleic acid was also considered and addressed by evaluating any alteration in the cycle threshold 
$(\mathrm{Ct})$ values as a surrogate marker for viral load in the collected specimens with and without lignocaine.

\section{Methodology}

\section{Objectives}

The primary objective of this study was to evaluate any significant decrease in patient discomfort during oropharyngeal sampling for COVID-19 with the use of topical lignocaine application using a lozenge. The secondary objective was to check if topical lignocaine use led to deterioration of sample quality by altering the $\mathrm{Ct}$ values and hence causing a falsenegative result.

\section{Study design and setting}

This prospective cohort study was conducted in May-June 2020 at a dedicated COVID-19 quarantine facility attached to our tertiary care institute. Ethical clearance was obtained from the institute ethics committee (Ref IEC-251/4/2020 "Documents of the study are approved from ethical angle prospectively w.e.f. 24th April 2020"). All patients provided written informed consent for enrollment in the study.

\section{Patient population}

As this was an exploratory study, a sample size of 40 was chosen. Ambulatory patients, who had tested positive on the qualitative reverse transcription RT-PCR for detection of SARS-CoV-2 RNA, which was conducted on specimens drawn using combined oropharyngeal and nasal swabs as per our institutional policy, were considered for inclusion in the study. Patients aged $<18$ years, pregnant or lactating women, patients with a documented history of hypersensitivity to lignocaine, patients with severe disease (room air oxygen saturation $<90 \%$ ) and those refusing to participate were excluded. Those whose symptom profile changed within $48 \mathrm{~h}$ of the initial sample were also excluded to avoid confounding.

\section{Sample collection procedure}

An oropharyngeal swab was performed within $48 \mathrm{~h}$ of the first swab sample, which had tested positive. Unlike the first pharyngeal swab (Standard, sample A) the second swab was taken after achieving topical lignocaine anaesthesia with a $200 \mathrm{mg}$ lignocaine lozenge (Xynova ${ }^{\circledR}$ ). This lozenge is mounted on a soft plastic stick mimicking a lollipop and the patients were instructed to suck it for 3-4 min. The repeat oropharyngeal swab was taken by single investigator $[\mathrm{AK}]$ for all recruited patients after 3-5 min of the local anaesthetic application, using a wooden disposable tongue depressor and polyester swab. This was then coupled with the standard nasal swab from both sides of the nasal cavity using a flexible soft polyester swab, to achieve matching of these samples (sample B) with their controls (sample A). The specimens (sample B) were then placed in $3 \mathrm{ml}$ of viral transport medium (HiMedia Laboratories, India) and transported in a triple-layered packaging in an ice-box to the institute's biosafety level 2 laboratory (BSL 2). Patients were asked to rate their discomfort on a visual analog scale (VAS) of 1-10 where 1 represented no discomfort and 10 meant intolerable discomfort. They were also asked in a 'yes or no' format whether the usage of lignocaine made the sample collection more comfortable for them. They were instructed to not drink or eat for $30 \mathrm{~min}$ following the procedure for the risk of aspiration and were observed for any lignocainerelated complications for an hour after the procedure.

\section{Laboratory procedure}

Sample A of the patients who had tested positive was preserved at $-80^{\circ} \mathrm{C}$. Both sample A and B were then tested as per usual procedure by qualitative real-time RT-PCR for detection of SARS-CoV-2 RNA. All the samples were processed in a class II, type A2 biological safety cabinet using BSL2 work practices. Two $500 \mu \mathrm{l}$ aliquots of each specimen were distributed to storage vials and stored at $-80^{\circ} \mathrm{C}$ until testing.

\section{RNA extraction and real-time RT-PCR assay}

Each pair of clinical samples (sample A and B) was put up in the same run for RT-PCR. RNA was extracted from the clinical samples using the automated nucleic acid extractor MagMAX ${ }^{\mathrm{TM}}$ Express-96 system (Applied Biosystems, Thermo Fisher Scientific, USA). RNA was reverse transcribed and amplified by using an FDA-EUA approved, Indian Council of Medical Research (ICMR) recommended Real-Time One-Step RT-PCR Master Mix (Shanghai Fosun, China) in the Agilent AriaMx Real-Time PCR system for detection of SARS-CoV-2. This kit amplifies conserved sequences from the ORF (Open Reading Frame) 1ab gene, N (Nucleocapsid encoding) gene, and $\mathrm{E}$ (Envelope encoding) gene of SARSCoV-2. Real- time RT-PCR tested the SARS-CoV-2 specific ORF gene (present at the $5^{\prime}$ end of the viral genome) with either $\mathrm{N}$ or $\mathrm{E}$ gene detection, both of which are present at the $3^{\prime}$ end of the viral genome. The $\mathrm{Ct}$ values of the ORF $1 \mathrm{ab}$ gene were used for analysis in this study.

\section{Statistical analysis}

Ct values of the RT-PCR of the two groups- without lignocaine (sample A) and with lignocaine (sample B) were 
compared for any statistical significance using the paired t-test and checked for any statistically significant difference ( $p$ value of $<0.05$ ). Wilcoxon signed rank test was used to compare the discomfort scores on VAS. $p$ value of $<0.05$ was considered significant. Statistical analysis was conducted using SPSS ${ }^{\circledR}$ version 24.0.

\section{Results}

Forty adult patients were included in the study. Clinicodemographic profile is tabulated in Table 1. Only six patients were asymptomatic. Sore throat and cough were the most commonly reported symptoms.

Our outcomes have been tabulated in Table 2. When asked about the comfort, 29 out of 40 patients (72.5\%) favoured the use of lignocaine over previous procedure. This was largely attributed to blunting of the gag and cough

Table 1 Clinico-demographic characteristics of the patient population

\begin{tabular}{ll}
\hline Characteristics & $\begin{array}{l}\text { Number of } \\
\text { patients, } n \\
(\%)\end{array}$ \\
\hline Sex & \\
Male & $28(70 \%)$ \\
Female & $12(30 \%)$ \\
Age (in years) & \\
Mean \pm SD & $35.9 \pm 10.88$ \\
Time to repeat samples & \\
$12-24$ h & $16(40 \%)$ \\
$24-48$ h & $24(60 \%)$ \\
Predominant symptom & \\
Asymptomatic & $6(15 \%)$ \\
Anosmia & $4(10 \%)$ \\
Rhinorrhea & $9(22.5 \%)$ \\
Cough & $20(50 \%)$ \\
Sore throat & $25(62.5 \%)$ \\
Nasal obstruction & $5(12.5 \%)$ \\
Dysgeusia & $4(10 \%)$ \\
Fever & $13(32.5 \%)$ \\
Bodyache & $9(22.5 \%)$ \\
Comorbidities & \\
No comorbidities & $34(85 \%)$ \\
Diabetes & $3(7.5 \%)$ \\
Hypertension & $2(5 \%)$ \\
Hypothyroidism & $1(2.5 \%)$ \\
NEWS Score & \\
0 & $30(75 \%)$ \\
2 & $10(25 \%)$ \\
& 0 \\
\hline & \\
& \\
&
\end{tabular}

${ }^{a}$ National Early Warning Score indicating the severity of illness of a patient, a higher score depicts a more severe disease
Table 2 Primary and secondary outcomes of our study

\begin{tabular}{ll}
\hline Outcomes & $\begin{array}{l}\text { Number of } \\
\text { patients, } n \\
(\%)\end{array}$ \\
\hline $\begin{array}{l}\text { Patients reporting sample } \mathrm{B}^{\mathrm{a}} \text { as more comfortable } \\
\text { Median (IQR) VAS scores (for discomfort) }\end{array}$ & $29(72.5 \%)$ \\
Sample A $^{\mathrm{b}}$ & $7(1)$ \\
Sample B & $5(2)$ \\
Mean Ct values \pm SD & \\
Sample A & $17.21 \pm 5.25$ \\
Sample B & $18.44 \pm 4.8$ \\
\hline
\end{tabular}

VAS Visual Analog Scale, $C t$ Cycle threshold

${ }^{\text {a }}$ Swab sample taken after lignocaine application

${ }^{\mathrm{b}} \mathrm{S} w a b$ sample taken without lignocaine application

reflex. The median (IQR) VAS score for discomfort also dropped from 7(1) (range 5-9) when swabs were taken without lignocaine to 5(2) (range 3-8) when lignocaine was used $(p<0.00001)$ implying a significantly increased comfort level. There was no observed sampling related complication in either group. All positive samples were picked up by sample $\mathrm{B}$. Mean $\mathrm{Ct}$ value $\pm \mathrm{SD}$ for the 'sample A' group was $17.21 \pm 5.25$ while it was $18.44 \pm 4.8$ for the 'sample B' (Lignocaine group). There was no statistically significant difference between the two groups $(p=0.11)$.

\section{Discussion}

This study demonstrated that the use of oropharyngeal lignocaine during sample collection for SARS CoV-2 RT-PCR is associated with reduced patient discomfort without compromising the sample quality. To the best of our knowledge, this is the first study to demonstrate these findings in patients with COVID-19.

Samples collected for real-time RT-PCR for the detection of SARS-CoV-2 require rubbing of the swabs on the oropharyngeal mucosa to ensure that adequate number of cells is obtained for testing. High aerosol generation is an obvious consequence of airway reactivity, and places the $\mathrm{HCW}$ to an undue risk of acquiring infection while sampling. Most of the HCWs involved in sample collection for COVID-19 currently use personal protective equipment (PPE) to protect themselves against this aerosol. Multiple ideas, some of them novel, are being employed worldwide to curtail the generation of aerosols. Using nasal swabs instead of nasopharyngeal swabs, samples from gargled fluids and saliva, using glass-walled kiosks, sampling from the side using a mirror for guidance, self-sampling by the patient and sampling in the patient's car through the side window have been employed but mitigate the technique having direct 
impact on sample quality [3-6]. Through this study we wish to introduce lignocaine as a possible measure that improves patient comfort and hence, reduces aerosol while sampling.

Topical lignocaine, in the form of lozenges has been used routinely by Otolaryngologists and gastroenterologists for the purpose of anaesthesia in uncomfortable upper aerodigestive procedures with documented safety and effectiveness $[7,8]$. Majority of our patient population $(72.5 \%)$ reported significantly decreased discomfort during the process of sampling as revealed by the improved median VAS scores. Wilson et al. have reported that the explosive velocities generated during coughing and sneezing lead to shearing forces over the airways producing a high viral load expulsion and should be curtailed as much as possible [9]. Viral particles may stay suspended in air for variable periods making decontamination of the area difficult and transmission risk higher. Anderson et al. have commented at length as how the airborne viral particles have the ability to travel distances $>1 \mathrm{~m}$ and play an important part in community transmission [10]. It is thus imperative that upper airway reflexes are minimised, an objective which the lignocaine lozenges were able to achieve. Even asymptomatic patients have been reported to transmit the disease and should be considered for topical lignocaine application [11].

Preservatives like cetylpyridinium chloride are used in lignocaine preparations, which have antimicrobial properties [12]. These facts prompted us towards a theoretical concern of tampered sample quality with lignocaine usage. Despite the presence of multiple confounding factors, e.g. the amount of cellular material obtained during the collection, temporal variability in viral shedding over a course of 24-48 h, effect of the host immune response on the virus, the mean $\mathrm{Ct}$ values showed no statistically significant difference between the sample A group (mean 17.21) and sample B group (mean 18.44), demonstrating a non-significant effect of the medication on SARS-CoV-2 concentration in the obtained sample.

There are other described modalities to administer topical lignocaine, in the form of nebulisation and topical sprays. These methods cannot be favoured in the COVID-19 era because of the aerosol they generate. Williams et al. have reported the possibility of cross-infection due to use of a common nozzle on topical spray applicator [13]. Lozenges also provide ease of administration, and prevent cross-infection between two patients.

Usage of lignocaine may be particularly useful for HCWs and community at large to further enhance the safety of work and community environment, who already are at an increased risk of infection, especially while working in a community setting [14]. We believe that the results obtained from this study may be applicable to the other viruses (Influenza, SARS, Ebola) as well until any study is conducted dedicated to these particular viruses.
Our study had several limitations. First, we did not use lignocaine application in the nasal cavity and hence, cannot comment if the yield of the sample was contributed by the nasal swab alone or both the swabs. However, as we have already mentioned, application of lignocaine in nasal cavity via spray may itself lead to aerosol generation negating the purpose for which it is used. Also, the nasal swab can be collected by the operator from the side or by the patient herself, unlike the oropharyngeal swab which needs the operator to directly visualise the posterior pharyngeal wall and hence get exposed to the possible cough or gag reflex. Second, we did not evaluate for the operator-perceived aerosol generation with and without lignocaine usage, as multiple operators had been deployed for screening the COVID-19 suspects and collecting the first sample and usage of VAS brings in interoperator variability. Third, the patients were asked about their discomfort score after 'Sample B' which brings in recall bias to the results. Fourth, we enrolled only the patients who tested positive for SARS-CoV-2, thereby allowing selection bias in our study population. However, it was done to achieve the secondary objective while minimising resource use. Fifth, as the patients were not blinded to the usage of lignocaine, we cannot exclude a placebo effect contributing to the decrease in reported discomfort.

\section{Conclusion}

Topical oropharyngeal lignocaine application, while significantly increasing the comfort levels for the patient during oropharyngeal sampling for COVID-19, has no effect on the detection of SARS-CoV-2 in the upper airway (when oropharyngeal swab is combined with nasal swab), and thus should be considered as a measure to reduce the viral inoculum a HCW sampling a COVID-19 suspect gets exposed to.

Acknowledgements We would like to thank Vikas H, Shubhangi Khare, Salman Khan, Megha Brijwal, Smriti Panda and Pirabu Sakthivel for their help.

Funding Indian Council of Medical Research (ICMR).

\section{Compliance with ethical standards}

Conflict of interest The authors declare that they have no conflict of interest to disclose.

Informed consent An informed written consent was obtained from all participating patients included in the study.

\section{References}

1. Wang W, Xu Y, Gao R et al (2020) Detection of SARS-CoV-2 in different types of clinical specimens. JAMA. https://doi. org/10.1001/jama.2020.3786 (Published online March 11, 2020) 
2. van Doremalen N, Bushmaker T, Morris DH et al (2020) Aerosol and surface stability of SARS-CoV-2 as compared with SARSCoV-1. N Engl J Med. https://doi.org/10.1056/NEJMc2004973 (Published online March 17, 2020)

3. To KK-W, Tsang OT-Y, Leung W-S et al (2020) Temporal profiles of viral load in posterior oropharyngeal saliva samples and serum antibody responses during infection by SARS-CoV-2: an observational cohort study. Lancet Infect Dis. 20(5):565-574. https:// doi.org/10.1016/S1473-3099(20)30196-1

4. Saito M, Adachi E, Yamayoshi S et al (2020) Gargle lavage as a safe and sensitive alternative to swab samples to diagnose COVID-19: a case report in Japan. Clin Infect Dis. https://doi. org/10.1093/cid/ciaa377 (Published online April 2020)

5. Pasomsub E, Watcharananan SP, Boonyawat K et al (2020) Saliva sample as a non-invasive specimen for the diagnosis of coronavirus disease 2019: a cross-sectional study. Clin Microbiol Infect. https://doi.org/10.1016/j.cmi.2020.05.001 (Published online 2020)

6. Tu Y-P, Jennings R, Hart B et al (2020) Swabs collected by patients or health care workers for SARS-CoV-2 testing. N Engl J Med. https://doi.org/10.1056/NEJMc2016321 (Published online June 2020)

7. Mogensen S, Treldal C, Feldager E et al (2012) New lidocaine lozenge as topical anesthesia compared to lidocaine viscous oral solution before upper gastrointestinal endoscopy. Local Reg Anesth 5:17-22. https://doi.org/10.2147/LRA.S30715

8. Salale N, Treldal C, Mogensen S et al (2014) Bupivacaine lozenge compared with lidocaine spray as topical pharyngeal anesthetic before unsedated upper gastrointestinal endoscopy: a randomized controlled trial. Clin Med Insights Gastroenterol 7:55-59. https:// doi.org/10.4137/CGast.S18019
9. Wilson NM, Norton A, Young FP, Collins DW (2020) Airborne transmission of severe acute respiratory syndrome coronavirus-2 to healthcare workers: a narrative review. Anaesthesia. https://doi. org/10.1111/anae.15093 (Published online April 2020)

10. Anderson EL, Turnham P, Griffin JR, Clarke CC (2020) Consideration of the aerosol transmission for COVID-19 and public health. Risk Anal 40(5):902-907. https://doi.org/10.1111/risa.13500

11. Wang D, Hu B, Hu C et al (2020) Clinical characteristics of 138 hospitalized patients with 2019 novel coronavirus-infected pneumonia in Wuhan China. JAMA 323(11):1061-1069. https://doi. org/10.1001/jama.2020.1585

12. Mukherjee PK, Esper F, Buchheit K et al (2017) Randomized, double-blind, placebo-controlled clinical trial to assess the safety and effectiveness of a novel dual-action oral topical formulation against upper respiratory infections. BMC Infect Dis 17(1):74. https://doi.org/10.1186/s12879-016-2177-8

13. Williams OA, Wilcox MH, Nicol CD, Spencer RC, Reilly CS (1993) Lignocaine spray applicators are a potential source of cross-infection in the anaesthetic room. Anaesthesia 48(1):61-62. https://doi.org/10.1111/j.1365-2044.1993.tb06795.x

14. Lai X, Wang M, Qin C et al (2020) Coronavirus disease 2019 (COVID-2019) infection among health care workers and implications for prevention measures in a tertiary hospital in Wuhan China. JAMA Netw Open 3(5):e209666-e209666. https://doi. org/10.1001/jamanetworkopen.2020.9666

Publisher's Note Springer Nature remains neutral with regard to jurisdictional claims in published maps and institutional affiliations. 